\title{
A NOVEL MODULATION STRATEGY TO INCREASE STIMULATION DURATION IN NEUROMUSCULAR ELECTRICAL STIMULATION
}

\author{
RYAN J. DOWNEY, BSc, ${ }^{1}$ MATTHEW BELLMAN, BSc, ${ }^{1}$ NITIN SHARMA, PhD, ${ }^{1}$ QIANG WANG, PhD, ${ }^{1}$ \\ CHRIS M. GREGORY, PhD, PT, ${ }^{2}$ and WARREN E. DIXON, PhD ${ }^{1}$ \\ ${ }^{1}$ Department of Mechanical and Aerospace Engineering, University of Florida, Gainesville, Florida 32611-6250, USA \\ ${ }^{2}$ Brain Rehabilitation Research Center, North Florida/South Georgia Veterans Health System Department of Physical Therapy, \\ University of Florida, Gainesville, Florida, USA \\ Accepted 27 January 2011
}

ABSTRACT: Introduction: Neuromuscular electrical stimulation (NMES) has been shown to be an effective treatment for muscular dysfunction. Yet, a fundamental barrier to NMES treatments is the rapid onset of muscle fatigue. The purpose of this study is to examine the effect of feedback-based frequency modulation on the closed-loop performance of the quadriceps during repeated dynamic contractions. Methods: In the first experiment, subjects completed four different frequency modulation NMES protocols utilizing the same amplitude modulation control to compare the successful run times (SRTs). A second experiment was performed to determine the change in muscle response to high- and low-frequency stimulation. Results: Compared with constant-frequency stimulation, results indicate that using an error-driven strategy to vary the stimulation frequency during amplitude modulation increases the number of successful contractions during non-isometric conditions. Conclusion: Simultaneous frequency and amplitude modulation increases the SRT during closed-loop NMES control.

Muscle Nerve 44: 382-387, 2011

The use of electric current to activate skeletal muscle, often referred to as neuromuscular electrical stimulation (NMES), is commonly used in rehabilitation settings. The goal of NMES is to produce muscle contractions that aid in the recovery of strength, size, and function of human skeletal muscles to obtain physiological and functional benefits. Two primary applications for NMES include: (1) rehabilitation of skeletal muscle size and function via plastic changes in the neuromuscular system ${ }^{1-3}$; and (2) activation of muscle(s) to elicit movements that result in functional performance (i.e., standing, stepping, reaching, etc.), ${ }^{4-6}$ termed functional electrical stimulation (FES). NMES has been shown to be an effective treatment for muscular dysfunction in clinical cohorts such as stroke and spinal cord injury, ${ }^{4-6}$ although the specifics of how to optimize NMES delivery are not well established.

Methods are sought to increase the intensity and duration of rehabilitative treatments to more rapidly return affected individuals to activities of

Abbreviations: ANOVA, analysis of variance; FES, functional electrical stimulation; LEM, leg extension machine; NMES, neuromuscular electrical stimulation; RISE, robust integral of the sign of the error; RMS, root mean square; SRT, successful run time

Key words: amplitude modulation, frequency modulation, functional electrical stimulation, muscle fatigue, neuromuscular electrical stimulation Correspondence to: W. E. Dixon; e-mail: wdixon@.ufl.edu

(C) 2011 Wiley Periodicals, Inc.

Published online 15 August 2011 in Wiley Online Library (wileyonlinelibrary. com). DOI 10.1002/mus.22058 daily living and thereby reduce secondary complications and healthcare costs. A fundamental barrier to NMES/FES treatments is the rapid onset of muscle fatigue during repeated contractions. The onset of muscle fatigue during electrical stimulation is strongly correlated with stimulation parameters such as intensity, frequency, and pattern of stimulation. ${ }^{7}$ Contraction intensity is modulated by recruitment of additional motor units (regulated through stimulation amplitude and/or pulse width) as well as rate coding (regulated by subtetanic increases in stimulation frequency). Repeated contractions of appropriate intensity will likely require stimulators that can optimally modulate these parameters. Research studies targeting new technologies to reduce NMES-associated fatigue have achieved moderate success; however, a continued open research question is how the stimulation parameters (i.e., parameters of the activation dynamics) can be most effectively modulated to reduce the onset of fatigue while eliciting desired muscle contractions.

Previous studies have shown that the rate and level of fatigue during NMES may accelerate in response to high stimulation frequencies and intensities. $^{8-12}$ In particular, minimizing stimulation frequency is beneficial to achieving a given absolute torque when fatigue is a concern, and thus there is a need to lower the rate coding while recruiting more motor units to produce a desired muscle force/joint torque. These studies emphasize the importance of stimulation frequency over intensity in causing fatigue. Moreover, one study ${ }^{9}$ indicated that future efforts aimed at optimizing FES design should focus on dynamic contractions to emphasize the stimuli necessary to elicit predictable excursions at desired velocities in an effort to meet the contractile demands necessary for functional movement. Dynamic contractions introduce additional complexity over isometric actions, and muscle-specific properties (e.g., length-tension, force-velocity) influence controller performance during these types of contractions. Accordingly, studies that incorporate dynamic contractions while targeting reduction of fatigue during electrical stimulation may allow for a more rapid translation of results to clinical application. 
The experiments described herein address the effects of simultaneous frequency and amplitude modulation quantified by successful run times (SRTs) when the leg is controlled to follow a desired trajectory. The purpose of this study is to examine the effect of feedback-based frequency modulation on the closed-loop performance of the quadriceps femoris muscle group during repeated dynamic contractions with the goal of enabling more successful contractions, quantified by limb position error. A recently developed NMES control method ${ }^{13}$ is implemented through amplitude modulation with constant pulse width and different constant-frequency settings. The respective results are compared with those obtained by implementing the same controller with amplitude modulation and a feedback-based varied-frequency modulation scheme that adjusts the frequency to maintain the limb position tracking error within a specified range.

\section{METHODS}

All testing was performed using an apparatus that consists of a custom computer-controlled stimulation circuit and a leg extension machine (LEM; Fig. 1). The LEM includes optical encoders to measure the angle between the femur and the tibia. The LEM allows seating adjustments to ensure that the rotation of the knee is about the encoder axis. A 4.5-kg (10 lb.) load was attached to the weight bar of the LEM, and a mechanical stop was used to prevent hyperextension. A computer was used to collect data from the encoders and execute a closed-loop RISE (robust integral of the sign of the error) controller. ${ }^{13}$ The RISE controller calculates the voltage required at any given time for a subject's knee joint/shank to follow a given desired trajectory. The desired trajectory was selected as a sinusoid ranging from $5^{\circ}$ to $35^{\circ}$ with a period of 1.5 seconds. This trajectory was selected because the frequency and range of motion closely matches knee joint excursions during gait. Voltage was applied across the quadriceps muscle group with two bipolar self-adhesive surface electrodes. The electrodes were placed over the distal-medial and proximal-lateral portions of the quadriceps femoris muscle group to maximize the number of stimulated muscle fibers.

Seven healthy men and women (age 29.5 \pm 8 years) participated in two sets of experiments utilizing NMES to follow a desired trajectory. Because the left and right legs of any given subject may have differing levels of strength and respond differently to electrical stimulation (i.e., require different controller gains), the left and right legs of each subject were treated as separate sets of data. Unique letters A-N correspond to each of the 14

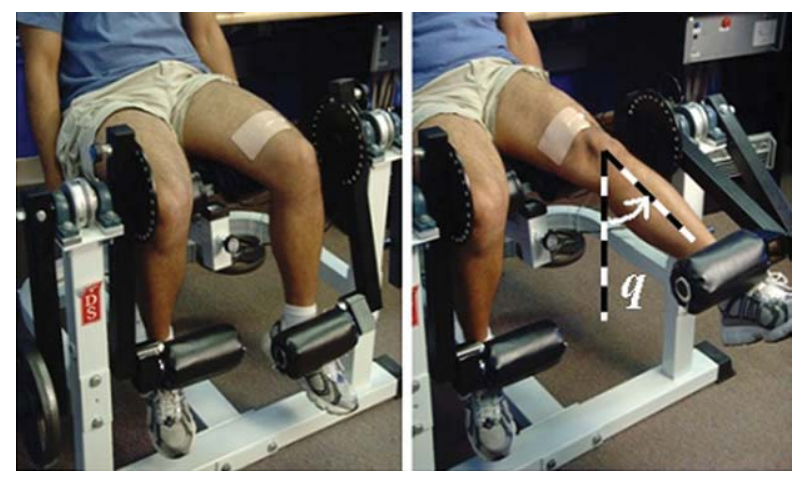

FIGURE 1. The experimental setup includes a leg extension exercise machine, encoders to determine the subject's leg angle, $\mathrm{q}(\mathrm{t})$, and a computer to control stimulation and gather data. [Color figure can be viewed in the online issue, which is available at wileyonlinelibrary.com.]

legs tested and are used in the Results section to depict which experiments each leg participated in. During testing, subjects were instructed to relax as much as possible and allow the stimulation to control the limb motion (i.e., the subject was not supposed to influence the leg motion voluntarily and was not allowed to see the desired trajectory). The electrical stimulation responses of healthy subjects have been reported to be similar to those of paraplegic subjects. ${ }^{14-17}$ Therefore, healthy subjects were used in NMES experiments as a substitute for affected individuals. Prior to participation, written informed consent was obtained from all subjects, as approved by the institutional review board at the University of Florida.

In the first experiment, for the same amplitude modulated control implementation, subjects completed four different frequency modulation NMES protocols including: constant $20 \mathrm{~Hz}$ (Protocol 1); constant $40 \mathrm{~Hz}$ (Protocol 2); decreasing from 40 $\mathrm{Hz}$ to $20 \mathrm{~Hz}$ (Protocol 3); and increasing from 20 $\mathrm{Hz}$ to $40 \mathrm{~Hz}$ (Protocol 4). SRTs were computed for each protocol by taking the total stimulation duration and subtracting the time spent in unsuccessful stimulation where unsuccessful stimulation is defined as any leg period when the root-meansquare (RMS) error is at least $2^{\circ}$ greater than the baseline error. Baseline error is defined as the RMS tracking error observed at the beginning of the protocol (i.e., before the onset of fatigue). To remove transient error from the baseline calculation, the first two periods of stimulation are neglected. The RMS error of the next three leg periods is calculated and saved as the baseline error. The SRT data were also used to compare the two varied-frequency protocols (Protocols 3 and 4$)$ at $10-\mathrm{Hz}$ intervals.

A second set of experiments was performed to determine the change in muscle response to highand low-frequency stimulation (i.e., low- or high- 
frequency fatigue). For each leg and each experiment, the protocols were completed in random order. To reduce the possibility that fatigue affected the results, a minimum 24-hour rest was required between each protocol.

Leg angle was controlled using the closed-loop nonlinear control strategy ${ }^{13}$ to adjust the voltage (i.e., amplitude modulation). To reduce the effect of day-to-day variability in muscle response to stimulation, the control gains were adjusted to minimize the baseline tracking error. Although the effect of control gain tuning was not studied in this research, efforts were made to reduce the possible influence of different gain tuning. Specifically, normalized tuning was used such that for each stimulation protocol, the controller was tuned for the middle frequency (i.e., $30 \mathrm{~Hz}$ ). If the control gain had a positive effect on the SRT, an extended time at this stimulation frequency would be expected, but no such effect was found. In addition to gain tuning, a pre-trial test was performed to determine the appropriate initial voltage to reduce the transient error.

Once the control gains and initial voltage level were determined, an algorithm was used to compute a baseline error. The algorithm neglected the first two periods of the stimulation (i.e., the first 3 seconds) to remove transient error from the calculation. The RMS error of the next three periods was calculated and determined to be the baseline error. For subsequent leg periods, the RMS error was calculated and compared with the baseline. For any period in which the RMS error was $2^{\circ}$ larger than the baseline, that period was determined to be unsuccessful.

For the varied-frequency protocols, each stimulation frequency was allowed two unsuccessful stimulations, and then the frequency was either decreased (Protocol 3) or increased (Protocol 4) by $2 \mathrm{~Hz}$. When the final stimulation frequency was reached (either $20 \mathrm{~Hz}$ or $40 \mathrm{~Hz}$, depending on the protocol), four unsuccessful stimulations were allowed before ending stimulation. For the constant-frequency protocols, four unsuccessful stimulations were allowed before ending stimulation. Unsuccessful stimulations were not required to be consecutive in determining when to modulate the stimulation frequency or end the stimulation. Unsuccessful stimulations were subtracted from the total stimulation time to calculate the SRT. By removing unsuccessful time, the experiments were comparable across both varied- and constant-frequency protocols.

In the second set of experiments, the varied-frequency protocols were examined to determine whether differences existed with respect to low- or high-frequency fatigue. Prior to and immediately following each protocol, 0.1 second of low-frequency $(10 \mathrm{~Hz})$ and 0.1 second of high-frequency $(100 \mathrm{~Hz})$ stimulation were delivered at a predetermined voltage to the quadriceps femoris muscle group. Voltage was determined in a pre-trial test such that either the evoked leg excursion was at least $25^{\circ}$ or the voltage reached $50 \mathrm{~V}$. The maximum leg angle reached by each stimulation was recorded. Changes in the maximum leg angle quantified the change in muscle response in terms of low- or high-frequency fatigue. Because the torque produced from $10-\mathrm{Hz}$ stimulation is significantly lower than that produced from $100-\mathrm{Hz}$ stimulation, the voltages were not set to be equal. ${ }^{10}$ However, the voltage supplied at each stimulation before the protocol began was equal to that supplied after the protocol.

A one-way analysis of variance (ANOVA) was performed on the normalized SRTs to ascertain if differences exist between the four protocols. To normalize the SRTs, each of the subject's SRTs obtained from the four protocols was divided by his/her maximum SRT. Normalization accounts for the variability in types of muscle between subjects (i.e., sprinter vs. marathon muscle). Post hoc analysis (Tukey-Kramer method) was used to determine differences between individual protocols. In addition, $t$-tests were used to determine differences between interval periods during the varied-frequency protocols as well as in change to muscle response to low- and high-frequency stimulation. The level of significance was set at $\alpha=0.05$ for all $t$-tests and post hoc analyses.

\section{RESULTS}

The SRT data are listed in Table 1 with the respective boxplots of the normalized data depicted in Figure 2. The results indicate that the mean normalized SRTs for Protocols 1 and 2 are statistically less than the mean normalized SRTs for Protocols 3 and 4 (Tukey-Kramer method, $\alpha=0.05$ ). However, no statistically significant differences were found between the two constant-frequency protocols or between the two varied-frequency protocols.

In addition to comparisons of the total SRTs between protocols, SRTs were also examined for both of the varied-frequency protocols in $10-\mathrm{Hz}$ stimulation frequency intervals. As indicated in Table 2, statistically more contractions were performed during the first interval $(20-30 \mathrm{~Hz})$ of Protocol 4 compared with the first interval $(40-30 \mathrm{~Hz})$ of Protocol $3(P=0.036)$. Similarly, normalized SRTs in the second interval $(30-20 \mathrm{~Hz})$ of Protocol 3 and the second interval $(30-40 \mathrm{~Hz})$ of Protocol 4 also suggest a statistical benefit to stimulation in the lower frequency interval $(P=0.006)$. 
Table 1. Successful run time (in seconds) of 12 healthy normal legs obtained when stimulated with four different protocols.

\begin{tabular}{|c|c|c|c|c|}
\hline & Protocol 1: & Protocol 2: & Protocol 3: & Protocol 4: \\
\hline Leg & $\begin{array}{c}20-H Z \\
\text { stim. freq. }\end{array}$ & $\begin{array}{c}40-\mathrm{HZ} \\
\text { stim. freq. }\end{array}$ & $\begin{array}{l}40-20-\mathrm{HZ} \\
\text { stim. freq. }\end{array}$ & $\begin{array}{l}20-40-H Z \\
\text { stim. freq. }\end{array}$ \\
\hline A & 121.5 & 61.5 & 318.0 & 241.5 \\
\hline B & 111.0 & 37.5 & 127.5 & 328.5 \\
\hline C & 18.0 & 57.0 & 88.5 & 66.0 \\
\hline $\mathrm{D}$ & 87.0 & 54.0 & 133.5 & 88.5 \\
\hline$E$ & 172.5 & 43.5 & 205.5 & 234.0 \\
\hline $\mathrm{F}$ & 54.0 & 40.5 & 133.5 & 82.5 \\
\hline$G$ & 43.5 & 87.0 & 547.5 & 97.5 \\
\hline $\mathrm{H}$ & 61.5 & 10.5 & 55.5 & 64.5 \\
\hline I & 100.5 & 48.0 & 67.5 & 159.0 \\
\hline J & 198.0 & 178.5 & 208.5 & 207.0 \\
\hline $\mathrm{K}$ & 147.0 & 48.0 & 136.5 & 166.5 \\
\hline $\mathrm{L}$ & 124.5 & 46.5 & 231.0 & 261.0 \\
\hline Mean & 103.25 & 59.38 & $187.75^{\star}$ & $166.38^{*}$ \\
\hline SD & 53.78 & 41.44 & 135.83 & 88.14 \\
\hline
\end{tabular}

*Significantly longer SRT than Protocol 1 and Protocol 2.

For the second set of experiments (Table 3), a statistically greater relative reduction in leg excursion occurred in response to high-frequency (100 $\mathrm{Hz})$ stimulation than to low-frequency $(10 \mathrm{~Hz})$ stimulation for both Protocol $3(P=0.006)$ and Protocol $4(P=0.017)$. No statistically significant difference was found between the two protocols at either $10 \mathrm{~Hz}$ or $100 \mathrm{~Hz}$.

\section{Discussion}

The onset of muscle fatigue during NMES/FES is strongly dependent on the stimulation parameters prescribed, such as intensity, frequency, and pattern of stimulation. ${ }^{7}$ Previous research by Binder-

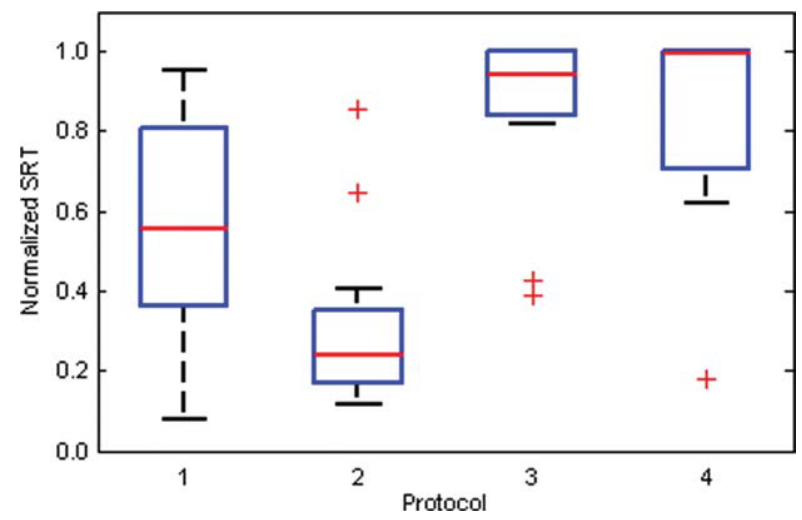

FIGURE 2. Boxplot obtained with ANOVA analysis performed on the normalized SRTs of 12 legs when they were stimulated with four different protocols. The F-value was obtained as 12.56. The $P$-value obtained was $4.59 \mathrm{E}-6$. The central marks in the boxes represent the median, the edges of the boxes represent the 25th and 75th percentiles, and the whiskers extend to the most extreme data points not considered to be outliers $( \pm 2.7 \sigma)$, as the outliers are plotted separately as plus signs. The median values for Protocols 1, 2, 3, and 4 are 0.555, 0.241, 0.943, and 0.996, respectively. [Color figure can be viewed in the online issue, which is available at wileyonlinelibrary.com.]
Table 2. Successful run time (in seconds) of 12 healthy normal legs computed in 10-HZ intervals for Protocols 3 and 4.

\begin{tabular}{lccccc}
\hline & \multicolumn{2}{c}{ Protocol 3 (freq. int) } & & \multicolumn{2}{c}{ Protocol 4 (freq. int.) } \\
\cline { 2 - 3 } \cline { 5 - 6 } Leg & $40-30 ~ H Z$ & $30-20 ~ H Z$ & & $20-30 ~ H Z$ & $30-40 ~ H Z$ \\
\hline$A$ & 114.0 & 214.5 & & 226.5 & 16.5 \\
B & 64.5 & 64.5 & & 312.0 & 19.5 \\
$\mathrm{C}$ & 78.0 & 25.5 & & 31.5 & 34.5 \\
$\mathrm{D}$ & 94.5 & 54.0 & & 88.5 & 0.0 \\
$\mathrm{E}$ & 162.0 & 48.0 & & 190.5 & 49.5 \\
$\mathrm{~F}$ & 40.5 & 93.0 & & 78.0 & 9.0 \\
$\mathrm{G}$ & 120.0 & 435.0 & & 88.5 & 13.5 \\
$\mathrm{H}$ & 54.0 & 7.5 & & 64.5 & 0.0 \\
$\mathrm{I}$ & 66.0 & 1.5 & & 142.5 & 19.5 \\
J & 178.5 & 30.0 & & 205.5 & 1.5 \\
K & 87.0 & 58.5 & & 162.0 & 6.0 \\
L & 135.0 & 175.5 & & 153.0 & 114.0 \\
Mean & 99.50 & $100.63^{*}$ & & $145.25^{\star}$ & 23.63 \\
SD & 43.25 & 123.43 & & 80.26 & 32.01 \\
\hline
\end{tabular}

$P$-values for one-tailed t-tests computed from normalized data (SRT was divided by each subject's maximum SRT). The t-tests were only used to compare the first interval of Protocol 3 to the first interval of Protocol 4 , and similarly to compare the second interval of each protocol. The first interval of one protocol should not be compared with the second interval of another protocol due to differing states of fatigue.

*Statistically significant difference vs. the corresponding interval of the other varied-frequency protocol.

Macleod et al., ${ }^{18}$ Kebaetse et al., ${ }^{19,20}$ and Kesar et al. ${ }^{21}$ indicates that constant high-frequency stimulation protocols produce more fatigue than constant low-frequency stimulation protocols, even at matched force levels, and that varied-frequency protocols have better performance than constantfrequency protocols. Fatigue levels have been shown to increase ${ }^{18}$ with increasing frequencies of 20,40 , and $60 \mathrm{~Hz}$ to achieve $20 \%$ or $50 \%$ of the maximum voluntary isometric contraction force. In addition, NMES has been applied ${ }^{19,20}$ to both healthy subjects and those with spinal cord injury to demonstrate that a varied-frequency strategy can improve the performance of repetitive non-isometric contractions. Better performance has been

Table 3. Percentage change in leg excursion angle when 10and $100-\mathrm{HZ}$ stimulation were applied to 6 legs before and after Protocols 3 and 4 were performed.

\begin{tabular}{|c|c|c|c|c|}
\hline \multirow[b]{3}{*}{ Leg } & \multicolumn{4}{|c|}{ Change in leg excursion angle (\%) } \\
\hline & \multicolumn{2}{|c|}{ Protocol 3} & \multicolumn{2}{|c|}{ Protocol 4} \\
\hline & 10-HZ stim. & 100-HZ stim. & 10-HZ stim. & 100-HZ stim. \\
\hline । & -22.71 & -36.53 & -76.79 & -93.48 \\
\hline J & -31.65 & -97.00 & -47.69 & -93.53 \\
\hline K & 25.64 & -74.20 & 19.03 & -80.63 \\
\hline $\mathrm{L}$ & -77.47 & -93.12 & -62.54 & -95.43 \\
\hline M & -32.50 & -84.14 & -13.85 & -71.92 \\
\hline $\mathrm{N}$ & 6.28 & -62.56 & 24.79 & -30.83 \\
\hline Mean & -22.07 & $-74.59^{\star}$ & -26.17 & $-77.64^{*}$ \\
\hline $\mathrm{SD}$ & 35.64 & 22.50 & 42.75 & 24.71 \\
\hline
\end{tabular}

$P$-values for one-tailed $t$-tests were computed.

*Statistically greater loss in excursion angle to $100-\mathrm{HZ}$ stimulation than to $10-\mathrm{HZ}$ stimulation. 
observed $^{19,20}$ by starting at a low stimulation frequency and later switching to a higher frequency than by applying either a constant low frequency or a high frequency alone. Isometric muscle performance has been demonstrated ${ }^{21}$ to improve by using a stepwise increase in stimulation frequency. Researchers have explored random modulation of the pulse frequency, amplitude, and pulse width to reduce fatigue ${ }^{22,23}$; however, random modulation was recently proven to be invalid. ${ }^{23}$ Doublet stimulation has also been demonstrated to provide both positive and negative effects on fatigue, depending on the protocol. ${ }^{24-27}$ In all the aforementioned studies, the stimulation intensity (amplitude) was kept constant, whereas frequency was modulated. Our study has examined the use of varied frequency and intensity stimulation to improve the closed-loop performance of the quadriceps femoris muscle group in terms of limb position tracking error. The results are more general in the sense that the intensity and frequency were modulated simultaneously while achieving successful task performance where the performance was measured by computing SRTs. They indicate that progressively increasing or decreasing the stimulation frequency yields statistically greater SRT (when compared with either constant high or constant low frequency). Although no statistically significant difference was found between the varied-frequency protocols (Protocol 3 and 4; Table 1) in terms of total SRT, further analysis of SRT intervals suggests statistical differences exist that favor the lower frequency intervals, independent of the initial frequency.

Few studies have attempted to demonstrate the effect of varying frequency from an initially high frequency to lower frequencies. One possible reason is the greater fatigue typically associated with high-frequency stimulation. Thus, it seems more reasonable to start with a low frequency and then change to a high frequency only when the muscle output may dictate higher demands. This may be especially true when stimulation intensity (or another stimulation parameter) is not modulated. In addition, this manner of frequency modulation (i.e., low to high) may more closely mimic biological approaches to muscle activation with the central nervous system prioritizing increases in recruitment versus rate coding during voluntary contractions. However, these results suggest that starting with a high frequency and then changing to a low frequency may also be considered a viable strategy for effective delivery of NMES.

Analysis of the two varied-frequency protocols suggests that the highest mean SRT occurred during the $20-30-\mathrm{Hz}$ interval. Specifically, the results show differences in the corresponding first and second intervals of both protocols, suggesting that the $20-30-\mathrm{Hz}$ interval may be a more preferable zone of stimulation, independent of fatigue state, during NMES control to delay the onset of fatigue. Although data from studies of frequency modulation alone (as opposed to frequency and intensity modulation) suggest a benefit in increasing frequency as muscles begin to fatigue, there are physiological mechanisms at the muscle level that may serve to help explain the current findings. Despite not being directly assessed in this study, the typical slowing of the muscle contraction (i.e., increased time to peak tension and half-relaxation times) that occurs in the fatigued state may make delivery of low-frequency stimuli later in protocols more efficient. During repeated contractions, a significant amount of energy is utilized for $\mathrm{Ca}^{++}$release/reuptake, and the combination of slowing of temporal characteristics with a lower activation frequency could result in a net benefit during this stage of the protocols. This would explain the improved performance during the lower frequency periods, initially in Protocol 3 but then in the final periods of Protocol 4. Importantly, these results suggest that there are significant benefits to simultaneously utilizing frequency and amplitude modulation instead of amplitude modulation alone for NMES control, as neither of the constant-frequency protocols was as successful as the varied-frequency protocols.

The second set of experiments was designed to compare the leg excursion angle elicited by the constant $10-\mathrm{Hz}$ and $100-\mathrm{Hz}$ stimulation before and after the varied-frequency protocols. The results suggest that there is no statistical difference in the mechanisms of fatigue (i.e., low-frequency vs. highfrequency fatigue) between the two protocols. These results suggest that both protocols have an equal effect on high-frequency fatigue (i.e., change in muscle response to high-frequency stimulation) and low-frequency fatigue (i.e., change in muscle response to low-frequency stimulation). As shown by Jones ${ }^{28}$ and the results of the present study, muscle fatigue can be caused by a number of electrophysiological and biological factors, but it is clear that the rate of fatigue is highly dependent on the frequency of stimulation. This study not only shows that the control performance can be improved in terms of maintaining the limb tracking error within a desired performance envelope by simultaneously modulating stimulation amplitude and frequency, but it also suggests a preferable stimulation frequency zone to delay the onset of fatigue.

In conclusion, our investigation has compared the performance of four modulation methods (constant low, constant high, increasing, and 
decreasing stimulation frequency). Although both varied-frequency stimulation protocols provided statistically significant improvements over constantfrequency stimulation, further research is required to determine whether there are other frequency modulation methods to further improve performance. Each modulation strategy fatigues muscle differently, leading to a diminished ability of the limb to perform a desired function. When compared with amplitude modulation alone, the variable amplitude and frequency modulation strategies in this study prolonged the ability of the limb to perform a desired function, quantified by SRT resulting from increased stimulation duration. Modulation strategies that lead to an increase in SRT are likely to improve FES task performance (e.g., an increased number of steps during walking or an increase in the duration of coordinated movement), because SRTs represent the duration of limb tracking within a specified RMS error. Importantly, although the dynamic contractions used in this study are likely more relevant than isometric contractions, it is critical for future studies to determine whether these same findings are indeed applicable during actual FES-induced activities. In addition, the underlying metabolic consequences of various stimulation strategies must also be examined to provide an understanding of the physiological mechanisms underlying improved performance during simultaneous frequency and amplitude modulation.

\section{REFERENCES}

1. Sabut SK, Sikdar C, Mondal R, Kumar R, Mahadevappa M. Restoration of gait and motor recovery by functional electrical stimulation therapy in persons with stroke. Disabil Rehabil 2010;32:1594-1603.

2. Thompson AK, Estabrooks KL, Chong S, Stein RB. Spinal reflexes in ankle flexor and extensor muscles after chronic central nervous system lesions and functional electrical stimulation. Neurorehabil Neural Repair 2009;23:133-142.

3. Yan T, Hui-Chan CW, Li LS. Functional electrical stimulation improves motor recovery of the lower extremity and walking ability of subjects with first acute stroke: a randomized placebo-controlled trial. Stroke 2005;36:80-85.

4. Hu XL, Tong KY, Li R, Chen M, Xue JJ, Ho SK, et al. Effectiveness of functional electrical stimulation (FES)-robot assisted wrist training on persons after stroke. Conf Proc IEEE Eng Med Biol Soc 2010;1: 5819-5822.

5. Kesar TM, Perumal R, Reisman DS, Jancosko A, Rudolph KS, Higginson JS, et al. Functional electrical stimulation of ankle plantarflexor and dorsiflexor muscles: effects on poststroke gait. Stroke 2009;40: 3821-3827.
6. Sabut SK, Lenka PK, Kumar R, Mahadevappa M. Effect of functional electrical stimulation on the effort and walking speed, surface electromyography activity, and metabolic responses in stroke subjects. J Electromyogr Kinesiol 2010;20:1170-1177.

7. Mizrahi J. Fatigue in muscles activated by functional electrical stimulation. Crit Rev Phys Rehabil Med 1997;9:93-129.

8. Binder-Macleod SA, Lee SCK, Baadte SA. Reduction of the fatigue induced force decline in human skeletal muscle by optimized stimulation trains. Arch Phys Med Rehabil 1997;78:1129-1137.

9. Gregory CM, Bickel CS, Sharma N, Dixon WE. Comparing the forceand excursion-frequency relationships in human skeletal muscle. Muscle Nerve 2008;38:1627-1629.

10. Gregory CM, Dixon WE, Bickel CS. Impact of varying pulse frequency and duration on muscle torque production and fatigue. Muscle Nerve 2007;35:504-509.

11. Matsunaga T, Shimada Y, Sato K. Muscle fatigue from intermittent stimulation with low and high frequency electrical pulses. Arch Phys Med Rehabil 1999;80:48-53.

12. Scott WB, Lee SC, Johnston TE, Binkley J, Binder-Macleod SA. Contractile properties and the force-frequency relationship of the paralyzed human quadriceps femoris muscle. Phys Ther 2006;86:788-799.

13. Sharma N, Stegath K, Gregory CM, Dixon WE. Nonlinear neuromuscular electrical stimulation tracking control of a human limb. IEEE Trans Neural Syst Rehabil Eng 2009;17:576-584.

14. Chang G-C, Lub J-J, Liao G-D, Lai J-S, Cheng C-K, Kuo B-L, et al. A neuro-control system for the knee joint position control with quadriceps stimulation. IEEE Trans Rehabil Eng 1997;5:2-11.

15. Hausdorff JM, Durfee WK. Open-loop position control of the knee joint using electrical stimulation of the quadriceps and hamstrings. Med Biol Eng Comput 1991;29:269-280.

16. Jezernik S, Wassink RGV, Keller T. Sliding mode closed-loop control of FES: controlling the shank movement. IEEE Trans Biomed Eng 2004;51:263-272.

17. Kurosawa K, Futami R, Watanabe T, Hoshimiya N. Joint angle control by FES using a feedback error learning controller. IEEE Trans Neural Syst Rehabil Eng 2005;13:359-371.

18. Binder-Macleod SA, Halden EE, Jungles KA. Effects of stimulation intensity on the physiological responses of human motor units. Med Sci Sports Exerc 1995;27:556-565.

19. Kebaetse MB, Binder-Macleod SA. Strategies that improve human skeletal muscle performance during repetitive, non-isometric contractions. Pflugers Arch 2004;448:525-532.

20. Kebaetse MB, Lee SC, Johnston TE, Binder-Macleod SA. Strategies that improve paralyzed human quadriceps femoris muscle performance during repetitive, nonisometric contractions. Arch Phys Med Rehabil 2005;86:2157-2164.

21. Kesar T, Chou L-W, Binder-Macleod SA. Effects of stimulation frequency versus pulse duration modulation on muscle fatigue. J Electromyogr Kinesiol 2008;18:662-671.

22. Graupe D, Suliga P, Prudian C, Kohn KH. Stochastically-modulated stimulation to slow down muscle fatigue at stimulated sites in paraplegics using functional electrical stimulation for leg extension. Neurol Res 2000;22:703-704.

23. Thrasher A, Graham GM, Popovic MR. Reducing muscle fatigue due to functional electrical stimulation using random modulation of stimulation parameters. Artif Organs 2005;29:453-458.

24. Bigland-Ritchie B, Zijdewind I, Thomas CK. Muscle fatigue induced by stimulation with and without doublets. Muscle Nerve 2000;23: $1348-1355$.

25. Karu ZZ, Durfee WK, Barzilai AM. Reducing muscle fatigue in FES applications by stimulating with N-let pulse trains. IEEE Trans Biomed Eng 1995;42:809-817.

26. Kebaetse MB, Turner AE, Binder-Macleod SA. Effects of stimulation frequencies and patterns on performance of repetitive, nonisometric tasks. J Appl Physiol 2002;92:109-116.

27. Thomas CK, Griffin L, Godfrey S, Ribot-Ciscar E, Butler JE. Fatigue of paralyzed and control thenar muscles induced by variable or constant frequency stimulation. J Neurophysiol 2003;89:2055-2064.

28. Jones DA. High- and low-frequency fatigue revisited. Acta Physiol Scand 1996;156:265-270. 